\title{
Role of Oxidative Stress in Various Stages of Psoriasis
}

\author{
Dipali P. Kadam • Adinath N. Suryakar • \\ Rajesh D. Ankush • Charushila Y. Kadam • \\ Kishor H. Deshpande
}

Received: 10 May 2009/Accepted: 30 December 2009/Published online: 24 September 2010

(C) Association of Clinical Biochemists of India 2010

\begin{abstract}
Psoriasis is a chronic inflammatory, proliferative skin disease characterized by pathological skin lesions due to various exogenous and endogenous factors. It is associated with a number of biochemical and immunological disturbances. Recently, it has been suggested that increased reactive oxygen species (ROS) production and compromised function of antioxidant system may be involved in the pathogenesis of this disease. In the present study, 90 psoriasis patients were selected. Disease severity was assessed by psoriasis area severity index score and grouped as mild, moderate and severe (each group consists of 30 subjects) and compared with 30 healthy controls. Serum levels of malondialdehyde, nitric oxide end products and the activities of antioxidant enzymes such as erythrocyte-superoxide dismutase, catalase and total antioxidant status were investigated in these groups/subjects. As compared to controls, we found severitywise significantly increased serum malondialdehyde, nitric oxide end products with decrease in erythrocyte-superoxide dismutase activity, catalase activity and total antioxidant status in patients with psoriasis suggesting worsening of the disease. It seems to be linked with the enhancement of Reactive Oxygen Species production and decreased antioxidant potential in psoriasis.
\end{abstract}

Keywords Psoriasis - Lipid peroxidation - Total antioxidant status · Oxidative stress · Enzymatic antioxidants

D. P. Kadam $(\bowtie)$ A. N. Suryakar · R. D. Ankush ·

C. Y. Kadam - K. H. Deshpande

Department of Biochemistry, Dr. V. M. Govt. Medical College,

Solapur, Maharashtra, India

e-mail: dip_6686@yahoo.co.in

\section{Introduction}

Psoriasis is a common chronic, inflammatory, proliferative skin disease that generally presents as chronic sharply demarcated, dull red, scaly plaques, particularly on extensor prominences and the scalp [1,2]. Skin cells mature and shed after about a month. However in psoriasis, the normal cycle of replacing old skin cells with new one becomes unbalanced [3].

The skin is a potential target for oxidative injury, as it is continuously exposed to UV radiation and other environmental stresses generating reactive oxygen species (ROS) [4]. ROS mediated oxidative damage involves a vast number of biological molecules since it causes lipid peroxidation, DNA modification, and secretion of inflammatory cytokines [5].

Plasma membranes of the skin cells in the psoriatic lesion have a significant increase in arachidonic acid, which is the natural substrate for synthesis of malondialdehyde (MDA), an end product of lipid peroxidation [6].

The keratinocytes, which make up the bulk of epidermis, constitutively express the neuronal isoform of Nitric Oxide Synthase (NOS I) whereas; the fibroblasts in the dermis and other cell types in the skin express the endothelial isoform (NOS III). Under certain conditions, virtually all skin cells appear to be capable of expressing the inducible NOS isoform (NOS II). The NO` liberated following UV irradiation plays a significant role in initiating melanogenesis, erythema and immunosuppression [7]. A comprehensive and integrated antioxidant defense mechanism of skin is crucial in protecting this organ from ROS [8].

One important line of defense is a system of enzymes, including glutathione peroxidase, superoxide dismutase (SOD) and catalase (CAT) which decrease the concentration of the most harmful oxidants [9]. A major contribution 
to the total antioxidants comes from antioxidant molecules in plasma. Antioxidants can protect the epidermis from the events that contribute to epidermal toxicity and diseases. Deficiencies in any of the antioxidant defense system can cause a reduction in the total antioxidant status (TAS) of an individual [3]. However, inadequate antioxidant protection or excess ROS production creates a condition known as a oxidative stress, contributing to the development of cutaneous disease and disorders [10].

Until recently, it was difficult to treat psoriasis, due to incomplete understanding of the factors behind pathogenesis of psoriasis which points out that there may be some lacuna in our understanding of etiopathology of psoriasis. Therefore, the present study was planned to investigate the possible involvement of lipid peroxidation-antioxidant status in psoriatic patients.

\section{Materials and Methods}

The present study was carried out in the Department of Biochemistry, Dr. V. M. Govt. Medical College, Solapur in collaboration with Shree Chhatrapati Shivaji Maharaj General Hospital, Solapur (Maharashtra).

A total of 120 individuals were included in this study, out of these 30 were healthy controls and 90 were newly diagnosed psoriasis patients aged between 20 to 60 years. Psoriasis patients were further graded according to the Psoriasis Area and Severity Index (PASI) [11], presenting at the time of blood collection as, mild (30 patients), moderate (30 patients) and severe (30 patients). The study protocol was approved by Ethical Committee of the institute.

The subjects who were alcoholics or smokers and had past or concurrent diseases like anemia, diabetes mellitus, cardiovascular diseases, liver or kidney diseases, inflammatory skin diseases which may possibly affect the redox status were excluded from the study. The psoriasis patients with any topical therapy within 4 weeks, systemic drug therapy or photo chemotherapy within 3 months were excluded from this study.

After obtaining prior consent, about $8 \mathrm{ml}$ of random blood was collected, of which 3-4 ml was poured into sterile plain bulb for estimation of MDA [12], $\mathrm{NO}^{\bullet}$ end products [13] and activity of CAT [14]. Remaining blood was collected in heparin bulb for estimation of erythrocyteSOD [15] and TAS [16].

All values were expressed as Mean \pm SD. The results obtained were analyzed statistically using the unpaired student's ' $z$ ' test, to evaluate the significance of differences between the mean values. $P$ values $<0.05$ were considered as significant. The strength of the association between the parameters was determined by the correlation coefficient analysis.

\section{Results and Discussion}

ROS induced oxidation of polyunsaturated fatty acids in biological system results in the formation of lipid peroxidation product MDA [10] which has been used as a biomarker of lipid peroxidation [17].

Present study shows that, serum MDA levels were significantly increased in mild $(P<0.01)$, moderate $(P<0.01)$ and severe $(P<0.01)$ psoriasis patients as compared with healthy controls (Table 1 ).

Furthermore, we found severity wise increase in MDA levels in these patients indicating that, the degree of elevation of serum MDA is associated with the progression of psoriasis.

ROS may be produced during the inflammatory process, in psoriasis, affecting primarily lipid metabolism of cells. Further, ROS that are produced by lipid peroxidation may activate phospholipase $\mathrm{A}_{2}$ and thus cause peroxidation of many mediators by arachidonic acid which finally metabolized to MDA [6, 10, 18].

Table 1 Shows changes in the levels of serum malondialdehyde (MDA), nitric oxide (NO ${ }^{\bullet}$ ) end products, activity of erythrocyte-superoxide dismutase (SOD), catalase (CAT) and total antioxidant status (TAS) in healthy control and different groups of psoriasis patients

\begin{tabular}{|c|c|c|c|c|}
\hline \multirow[t]{2}{*}{ Parameters } & \multirow[t]{2}{*}{ Healthy controls } & \multicolumn{3}{|l|}{ Psoriatic patients } \\
\hline & & Mild & Moderate & Severe \\
\hline Serum MDA (nmol/ml) & $1.54 \pm 0.19$ & $1.84 \pm 0.24 *$ & $3.11 \pm 0.24 *^{\bullet}$ & $4.35 \pm 0.45^{*}$ \\
\hline Serum $\mathrm{NO}^{\bullet}$ end products $(\mu \mathrm{mol} / \mathrm{l})$ & $35.49 \pm 6.31$ & $43.41 \pm 1.71 *$ & $50.85 \pm 3.68^{\bullet}$ & $62.24 \pm 4.20^{*}$ \\
\hline Erythrocyte-SOD (U/g of $\mathrm{Hb})$ & $1.84 \pm 0.19$ & $1.65 \pm 0.10^{*}$ & $1.30 \pm 0.07^{\bullet}$ & $0.98 \pm 0.08 *$ \\
\hline Serum CAT (KU/l) & $55.44 \pm 3.11$ & $48.56 \pm 2.53^{*}$ & $42.04 \pm 3.92^{\bullet}$ & $36.15 \pm 2.57 *$ \\
\hline Plasma TAS $(\mu \mathrm{mol} / \mathrm{l})$ & $966.66 \pm 33.89$ & $884.0 \pm 24.16^{*}$ & $798.33 \pm 28.87 *^{\bullet}$ & $740.33 \pm 18.52 *$ \\
\hline
\end{tabular}


Table 2 Correlation analysis of different parameters among the different groups of psoriasis patients

\begin{tabular}{llll}
\hline & Mild & Moderate & Severe \\
\hline MDA/NO & +0.98 & +0.93 & +0.95 \\
MDA/SOD & -0.99 & -0.95 & -0.93 \\
MDA/CAT & -0.99 & -0.95 & -0.91 \\
MDA/TAS & -0.97 & -0.93 & -0.94 \\
NO^/TAS & -0.97 & -0.98 & -0.97 \\
\hline
\end{tabular}

The skin is constantly exposed to oxidative stress induced by ROS that are generated both from endogenous neutrophils and external pro-oxidant stimuli [5]. Increased capacity for chemotaxis, adhesion [19] and increased ROS production in neutrophils [20], keratinocytes [21] and fibroblasts [22] have been reported in patients with psoriasis. Evidences show that increased oxidative stress in these patients as demonstrated by the high plasma MDA levels and compromised levels of the antioxidant defense enzymes are observed at the time of diagnosis itself [23].

Earlier reports suggest that, fibroblasts in the lesion-free skin of psoriasis patients show signs of increased oxidative damage even before the formation of characteristic psoriatic lesions which may be involved in the abnormal immune reactions leading to the onset of the disease [24].

As a potential regulator of keratinocyte growth and differentiation, the multifunctional signaling molecule $\mathrm{NO}^{\bullet}$ has been considered to be a strong candidate in the pathogenesis of psoriasis [25]. $\mathrm{NO}^{\bullet}$ is an important marker of inflammation [26].

In the present study, there was statistically significant increase in the levels of $\mathrm{NO}^{\bullet}$ end products in mild $(P<0.01)$, moderate $(P<0.01)$ severe $(P<0.01)$ psoriasis patients when compared with healthy controls. Further more, $\mathrm{NO}^{\bullet}$ end product levels in moderate psoriatic patients were significantly higher $(P<0.01)$ than the patients presenting with mild psoriasis, whereas, severe psoriatic patients exhibited significant rise in serum $\mathrm{NO}^{\circ}$ end products $(P<0.01)$ as compared to moderate psoriatics. We also found a positive correlation between MDA and $\mathrm{NO}^{\bullet}$ end products among the three different groups of psoriasis patients (Table 2). This finding shows extensive cohesiveness that, generation of $\mathrm{NO}^{\bullet}$ end products in excess may also trigger lipid peroxidation process.

Expression of iNOS is involved in the pathogenesis of cutaneous inflammation in psoriasis [27, 28]. Increase in mRNA expression of iNOS in skin lesions as compared to uninvolved skin have been reported [29]. Orem Asim et al. [30] observed that nitrite levels and nitrite-nitrate ratios appear to be good indicators for the increased $\mathrm{NO}^{\circ}$ production in patients and also showed a significant correlation with PASI.
In the present study we observed that, the erythrocyteSOD activities were significantly decreased in mild $(P<0.01)$, moderate $(P<0.01)$, severe $(P<0.01)$ psoriasis patients, as compared with healthy controls. Furthermore, erythrocyte-SOD activity in moderate psoriatic patients was significantly lower $(P<0.01)$ than the patients presenting with mild psoriasis, whereas, severe psoriatic patients exhibited significant decrease in erythrocyte-SOD activity $(P<0.01)$ as compared to moderate psoriatic patients. We also found a negative correlation between erythrocyte-SOD activity and serum MDA among the three different groups of psoriasis patients.

Our study indicates the possibility that, in the prediagnostic stage, serum antioxidants are low because they have been used in reducting inflammatory products. Decreased SOD activity might be related to epidermal hyper proliferation, because the ROS are thought to induce cell proliferation in various cell systems [31, 32].

Increased oxygen metabolism has been described in the psoriatic hyperproliferative epidermis, which depends on cutaneous blood flow. Increased $\mathrm{O}_{2}^{--}$production in the presence of decreased antioxidant activity would result in the accumulation of $\mathrm{H}_{2} \mathrm{O}_{2}$, which has an inhibitory effect on SOD activity [31, 33].

Since, the natural antioxidant defense system SOD limits the development of inflammatory and immune processes, we believe that, the tendency to decreased SOD activity in psoriasis patients is one of the reasons for the aggravated dermatological status.

One of the antioxidant enzyme capable of reducing hydrogen peroxide is CAT.

Present study showed that there was statistically significant decrease in the activity of catalase in mild $(P<0.01)$, moderate $(P<0.01)$ and severe $(P<0.01)$ psoriasis patients when compared to healthy controls. Furthermore, serum CAT activity in moderate psoriatic patients were significantly lower $(P<0.01)$ than the patients presenting with mild psoriasis, whereas, severe psoriatic patients exhibited significant decrease in CAT activity $(P<0.01)$ as compared to moderate psoriatic patients. We also found a negative correlation between CAT activity and serum MDA among the three different groups of psoriasis patients.

The CAT enzyme is most susceptible to the effect of UV radiation in the epidermis. Its activity can be affected by high peroxide concentration and by visible light [24]. Keratinocytes generate the $\mathrm{O}_{2}^{--}$is known to inhibit CAT. Furthermore, inactivation of SOD would lead to build-up of $\mathrm{H}_{2} \mathrm{O}_{2} \cdot \mathrm{H}_{2} \mathrm{O}_{2}$ generated through other processes have also been reported to induce CAT inactivation [33]. This might be the reason behind why we found a negative correlation between MDA and CAT. 
In vivo antioxidant status can be assessed by measuring individual plasma or tissue levels of antioxidants. Measuring the levels of these specific antioxidant molecules can yield valuable information, and low levels of such antioxidants provide suggestive, but not definitive, evidence of oxidative stress. However, determining total antioxidant capacity provides an index of the sum of the activities of all antioxidants [3].

In this study we found that, the plasma TAS levels were significantly decreased in mild $(P<0.01)$, moderate $(P<0.01)$ and severe $(P<0.01)$ psoriasis patients as compared with healthy controls. Further more, the TAS levels in moderate psoriatic patients were significantly lower $(P<0.01)$ than the patients presenting with mild psoriasis, whereas, severe psoriatic patients exhibited significant decrease in plasma TAS levels $(P<0.01)$ as compared to moderate psoriatics.

We further found, the significant negative correlation between plasma TAS and serum MDA levels among the three different groups of psoriasis. This correlation signifies that, decreased TAS may increase lipid peroxidation which may be a consequence of sustained decreased TAS.

We also found that significant negative correlation between plasma TAS and serum $\mathrm{NO}^{\bullet}$ end product levels among the three different groups of psoriasis. Since all NOS isoforms generate $\mathrm{O}_{2}^{\circ-}$ and mRNA expression of iNOS was markedly increased in skin lesions, there is increased production of $\mathrm{O}_{2}^{\bullet-}$ in the keratinocytes. This is the reason why we found the negative correlation between $\mathrm{NO}^{\bullet}$ end product and TAS among different groups of psoriasis.

The decreased TAS may be possibly due to depressed state of antioxidant system or due to the exaggerated inflammatory processes and oxidative stress in these patients. Antioxidants prevent oxidative injury of structural lipids and proteins contributing to barrier integrity, which is essential for healthy skin condition. This suggests that cellular redox environment plays a pivotal role in skin homeostasis and that skin disease could result from an imbalance between pro-oxidant and antioxidant stimuli [5].

In conclusion, this study provides an evidence for increased ROS production and decreased antioxidant defenses in psoriasis, reflected by increased lipid peroxidation and decreased TAS as well as decrease in SOD activity and $\mathrm{CAT}$ activities. Increased $\mathrm{NO}^{\bullet}$ end products in these patients may be a result of immunological and inflammatory mechanism which are important in etiopathogenesis of psoriasis. Further more we found worsened oxidant and antioxidant status according to the progression of the psoriatic lesions, which may be the crucial point in the pathogenesis of psoriasis and need further elucidations. Finally, of considerable interest is the possibility of using this information to develop novel strategies for diagnosis, prognosis and treatment of psoriasis patients are warranted.

\section{References}

1. Wozniak A, Drewa G, Krzyzynka-Malinowska E, Czajkowski R, Protas-Drozd F, Mila-Kierzenkowska C, et al. Oxidant antioxidant balance in patients with psoriasis. Med Sci Monit. 2007;13(1):R 30-3.

2. Vanizor KB, Orem A, Cimsit G, Yandi YE, Calapoglu M. Evaluation of the atherogenic tendency of lipids and lipoprotein content and their relationship with oxidant-antioxidant system in patients with psoriasis. Clin Chim Acta. 2003;328(1-2):71-82.

3. Luty-Frackiwicz A, Gorka IM, Januszewska L. Influence of smoking and alcohol consumption on total antioxidant status in patients with psoriasis. Adv Clin Exp Med. 2006;15(3):463-9.

4. Relhan V, Gupta SK, Dayal S, Pandey R, Lal H. Blood thiols and malondialdehyde levels in psoriasis. J Dermatol. 2002;29: 399-403.

5. Briganti S, Picardo M. Antioxidant activity, lipid peroxidation and skin diseases-what's new? JEADV. 2003;17:663-9.

6. Corrocher R, Ferrari S, Gironcoli M, Bassi A, Olivieri O, Guarini $P$, et al. Effect of fish oil supplementation on erythrocyte lipid pattern, malondialdehyde production and glutathione-peroxidase activity in psoriasis. Clin Chim Acta. 1989;179:121-32.

7. Cals-Grierson MM, Ormerod AD. Nitric oxide functions in the skin. Nitric Oxide. 2004;10(4):179-93.

8. Shindo Y, Witt E, Han D, Epstein W, Packer L. Enzymic and non-enzymic antoxidants in epidermis and dermis of human skin. J Investig Dermatol. 1994;102:122-4.

9. Langseth L. Oxidants, antioxidants and disease prevention. International Life Sciences Institute and ILSI Europe; 1995. p. 1-32.

10. Baz K, Cimen MYB, Kokturk A, Yazici AC, Eskandari G, Ikizoglu G, et al. Oxidant/antioxidant status in patients with psoriasis. Yonsei Med J. 2003;44(6):987-90.

11. Louden BA, Pearce DJ, Lang W, Feldman SR. A simplified psoriasis area severity index (SPASI) for rating psoriasis severity in clinical patients. Dermatol Online J. 2004;10(2):7.

12. Satoh K. Serum lipid peroxide in cerebrovascular disorders determined by a new colorimetric method. Clin Chim Acta. 1978;90:37-43.

13. Cortas NK, Wakid NW. Determination of inorganic nitrate in serum and urine by a kinetic cadmium reduction method. Clin Chem. 1990;36(8):1440-3.

14. Goth L. A simple method for determination of serum catalase activity and revision of reference range. Clin Chim Acta. 1991;196:143-52.

15. Das K, Samanta L, Chainy GBN. A modified spectrophotometric assay of superoxide dismutase using nitrite formation by superoxide radicals. Indian J Biochem Biophys. 2000;37:201-4.

16. Benzie IFF, Strain JJ. The ferric reducing ability of plasma [FRAP] as a measure of 'antioxidant power' the FRAP assay. Anal Biochem. 1996;239:70-6.

17. Lykkesfeldt J. Malondialdehyde as biomarker of oxidative damage to lipids caused by smoking. Clin Chim Acta. 2007;380:50-8.

18. Yildirim M, Inaloz HS, Baysal V, Delibas N. The role of oxidants and antioxidants in psoriasis. JEADV. 2003;17:34-6.

19. Popov I, Lewin G. A deficient function of the antioxidative system of the organism as an aetiopathogenetic factor in psoriasis. Med Hypotheses. 1991;35:229-36.

20. Miyachi Y, Niwa Y. Effects of psoriatic sera on the generation of oxygen intermediates by normal polymorphonuclear leucocytes. Arch Dermatol Res. 1983;275:23-6. 
21. Turner CP, Toye AM, Jones OTG. Keratinocyte superoxide generation. Free Radic Biol Med. 1998;24(3):401-7.

22. Raynaud F, Evain-Brion D, Gerbaud P, Marciano D, Gorin I, Liapi $\mathrm{C}$, et al. Oxidative modulation of cyclic AMP-dependent protein kinase in human fibroblasts: possible role in psoriasis. Free Radic Biol Med. 1996;22(4):623-32.

23. Hristakieva E, Gadjeva V. Seasonal variations in the activity of antioxidant enzymes and lipid peroxidation in psoriatic and vitiligo patients. Trakia J Sci. 2003;1(1):27-31.

24. Dimon-Gadal S, Gerbaud P, Therond P, Guibourdenche J, Anderson WB, Evain-Brion D, et al. Increased oxidative damage to fibroblasts in skin with and without lesions in psoriasis. J Investig Dermatol. 2000;114:984-9.

25. Tekin NS, Ilter N, Sancak B, Ozden MG, Gurer MA. Nitric oxide levels in patients with psoriasis treated with methotrexate. Mediat Inflamm. 2006;160431:1-5.

26. Clancy RM, Amin AR, Abramson SB. The role of nitric oxide in inflammation and immunity. Arthritis Rheum. 1998;41(7): 1141-51.

27. Kolb-Bachofen V, Fehsel K, Michel G, Ruzicka T. Epidermal keratinocyte expression of inducible nitric oxide synthase in skin lesions of Psoriasis vulgaris. Lancet. 1994;344:139.
28. Ormerod AD, Weller R, Copeland P, Benjamin N, Ralston SH, Grabowksi P, et al. Detection of nitric oxide and nitric oxide synthases in psoriasis. Arch Dermatol Res. 1998;290:3-8.

29. Sirsjo A, Karlsson M, Gidof A, Rollman O, Torma H. Increased expression of inducible nitric oxide synthase in psoriatic skin and cytokine-stimulated cultured keratinocytes. $\mathrm{Br} \mathrm{J}$ Dermatol. 1996;134(4):643-8.

30. Orem A, Aliyazicioglu R, Kiran E, Vanizor B, Cimnocodeit G, Deger O. The relationship between nitric oxide production and activity of the disease in patients with psoriasis. Arch Dermatol. 1997;133:1606-7.

31. Ilzuka H, Asaga H, Koike K, Ilzuka S. Decreased $\mathrm{Cu}, \mathrm{Zn}$ superoxide dismutase activity in psoriatic hyperproliferative epidermis. Eur J Dermatol. 1993;3:56-8.

32. Kobayashi T, Matsumoto M, Ilzuka H, Suzuki K, Taniguchi N. Superoxide dismutase in psoriasis, squamous cell carcinoma and basal cell epithelioma: an immunohistochemical study. $\mathrm{Br} \mathrm{J}$ Dermatol. 1991;124(6):555-9.

33. Gornicki A, Gutsze A. Erythrocyte membrane fluidity changes in psoriasis: an EPR study. J Dermatol Sci. 2001;27:27-30. 